\title{
Research of the spectral characteristics of healthy and fusarium-infected wheat seeds variety Moskovskaya 56 by hyperspectral spectroscopy
}

\author{
Maksim Moskovskiy ${ }^{1, *}$, Maksim Litvinov${ }^{1}$, Andrey Boiko², and Sergey Maklakov ${ }^{3}$ \\ ${ }^{1}$ Federal Scientific Agro-Engineering Center VIM, 1 Institutsky proezd, 5, Moscow, 109428, Russia \\ ${ }^{2}$ Don state technical university, Gagarin Square, 1, Rostov-on-Don, 344000, Russia \\ ${ }^{3}$ Rostov State Transport University, Rostovskogo Strelkovogo Polka Narodnogo Opolcheniya Square, \\ 2, Rostov-on-Don, 344038, Russia
}

\begin{abstract}
In this paper, a spectroscopic identification method is considered for determining the maxima and minima of spectral lines to identify pathogenic microflora in grain seeds. The paper presents the justification for the application of the method of hyperspectral imaging, in order to identify the disease fusarium in the seeds of soft winter wheat. Based on the graphs, it can see the general picture of the influence of the disease fusarium on wheat grains. There is a general decrease in the reflectivity of the grain surface. The strongest deviation of spectral lines is observed in the limit from $660 \mathrm{~nm}$ to $900 \mathrm{~nm}$.
\end{abstract}

\section{Introduction}

Hyperspectral imaging (HSI) combines the near infrared (NIR) and visible (RGB) spectra to provide information on the radiation intensity and the predominance of elements in the chemical composition.

Compared to narrowband multispectral cameras, hyperspectral cameras cover all spectral bands in a given range. As a result, the image is a hyperspectral cube, in which the $\mathrm{X}$ and $\mathrm{Y}$ axes represent the visualization of the photographed surface with metadata binding, the $\mathrm{Z}$ axis, in turn, can have a set of spectroscopic characteristics such as reflectivity, fluorescence, Raman spectrum, etc.

Fusarium disease is of fungal origin and occurs with the defeat of the ear by mycotoxins, which in turn makes the infected grain unsuitable for food use. Bread baked from flour obtained from severely affected grain has an intoxicating property and causes severe toxicosis, accompanied by indigestion, vomiting, loss of working capacity, etc. Poisoning of animals is also possible.

The relative simplicity of the formation of conidia allows the fungus to form a huge number of infectious structures in a short period of time. Under optimal conditions, after a few days, numerous conidia are formed on the spikelet scales, which serve as a new source

\footnotetext{
* Corresponding author: maxmoskovsky74@yandex.ru
} 
of plant infection. The period of appearance of a visible mass of conidia in the field depends on the time of inoculation, the susceptibility of plants, environmental conditions and is on average 5-10 days. Under favorable conditions for the fungus F. graminearum, it is able to form 3-4 generations during the flowering period - the milky-wax ripeness of the grain. In the form of mycelium, the fungus remains in seeds, on plant residues of cultivated crops and on wild weeds.

To assess the degree of infestation of an ear with Fusarium, a special scale is used, shown in Figure 1.

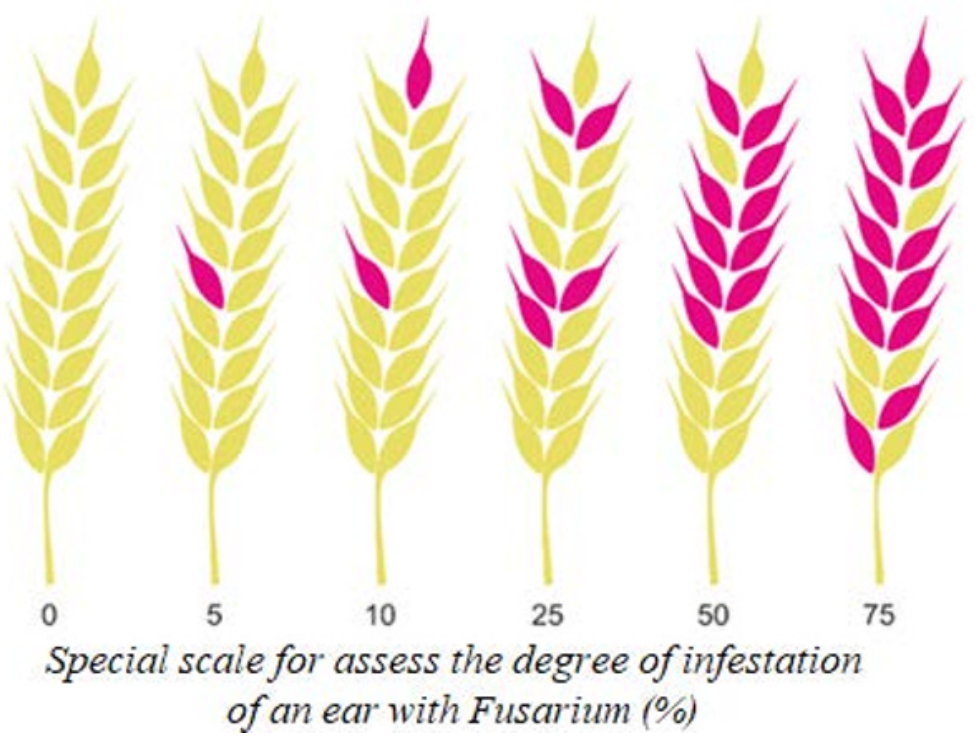

Fig. 1. Scale for assessing the infestation of the ear with Fusarium

\section{Materials and methods}

\subsection{Wheat samples for research of Fusarium infection.}

Winter wheat variety Moskovskaya 56 was used as a starting material for assessing the comparative assessment of the change in the reflectivity of the seed surface during infection on a multispectral image. The winter wheat variety Moskovskaya 56 has the following biological characteristics: Ear of medium length $(7.4 \mathrm{~cm})$, medium density $(18-19$ spikelets per $10 \mathrm{~cm}$ of the stem), medium awn, diverging. The average number of spikelets in an ear is $14-16$, of grains - 27-30. The mass of grain per ear is 1.06-1.26 g, the mass of 1000 grains is 39.6-45.3 g. Biological features. The variety is mid-season, ripens simultaneously with the Zarya standard. Winter hardiness is high, overwintering for 4 years - 94.4\%, and at Zarya $83.0 \%$, an excess of $11.4 \%$. The variety is distinguished by a large number of productive stems per $1 \mathrm{~m}^{2}$, an average of 564 stems over three years, which is 106 stems higher than the standard. Plant height $105 \mathrm{~cm}$, sturdy stem. In terms of lodging resistance, it exceeds the standard by 0.9 points. Resistant to brown rust and powdery mildew. Technological data. According to the competitive variety testing (2001-2003), the grain nature was $808 \mathrm{~g} / \mathrm{l}$, the protein content in the grain was $14.2 \%$, the raw gluten in the flour was $37.8 \%$, the flour strength was 251 e.a., the IDC index was 84 e. .shk., volumetric bread yield $993 \mathrm{~cm} 3$. For the Zarya variety, respectively, these indicators are 782 g / l, 15.4\%, 40.3\%, 390 units, 86 units, $1032 \mathrm{~cm}^{3}$. 
The selected sample was the harvest of 2020, the Central Black Earth zone of Russia. As samples for photographing, 6 weighed portions of seeds, 1000 grains each, were taken. Of the 6 samples, three are infected with Fusarium with a $72 \%$ lesion rate.

\subsection{Equipment}

Specim IQ hyperspectral camera (Spectral Imaging Ltd., https://www.specim.fi/iq/) was used as the equipment for the research. The camera allows evaluating the reflection spectra in the range $400-1000 \mathrm{~nm}$. The spectral resolution of the camera is $7 \mathrm{~nm}$ and includes 204 bands, the spatial resolution of the sensor is $512 \times 512$ pixels.

The technical characteristics of the camera are given in more detail in (Bohnenkamp et al., 2018). The camera was installed on a tripod above a table (Figure 2) taking into account that the supplied calibration plate takes up at least $10 \%$ of the entire image. The samples were illuminated with three $72 \mathrm{~W}$ luminescent panels. Before shooting, the camera was calibrated using a calibration panel to adjust the photosensitivity; after that, when taking a series of images, the panel was removed from the frame.

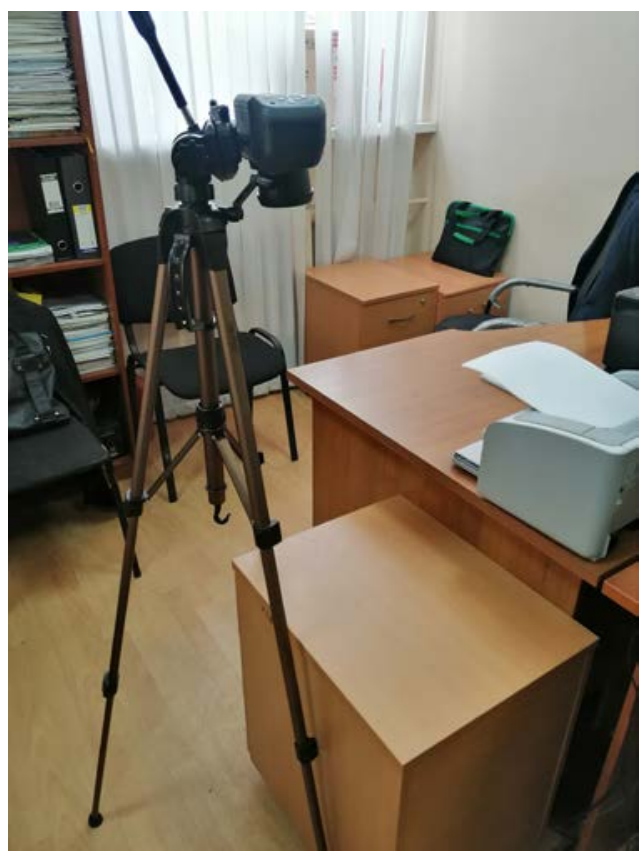

Fig. 2. Specim IQ camera on a tripod

\section{Method of Spectral image processing}

Preliminary visual quality control of hyperspectral images was carried out using the Albedo 4.0.23 software. This program is designed to determine the physical and chemical composition of an object by the pixels of the raster image. Each pixel is characterized by its own spectrum, decomposed into 200-1000 spectral channels.

Each image is analyzed in the following spectra: Near Red (NIR), Red, Green, Blue. To exclude foreign objects from the analysis field, polygons are highlighted in the studied images (Figure 3). The spectral line was calculated for each polygon. 

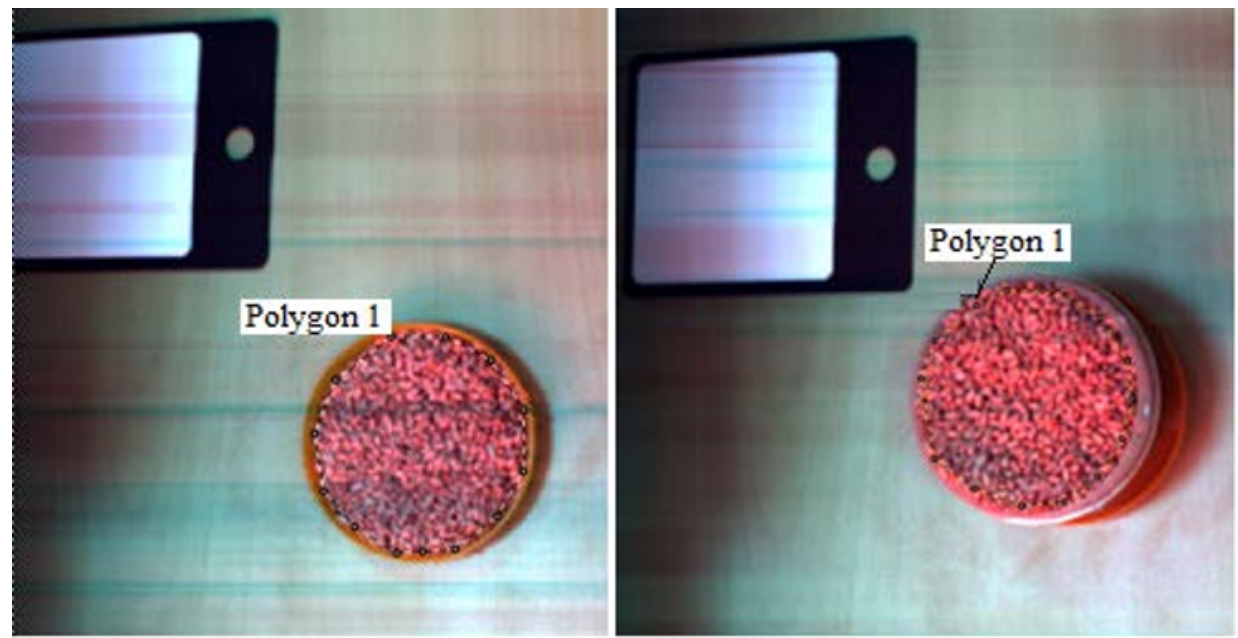

Fig. 3. Definition of the research area

In the graphs with the results of calculations (Figures 4,5), Bands are highlighted that can be excluded from subsequent analysis, since these areas reflect the absorption bands of water and oxygen present in the graph.

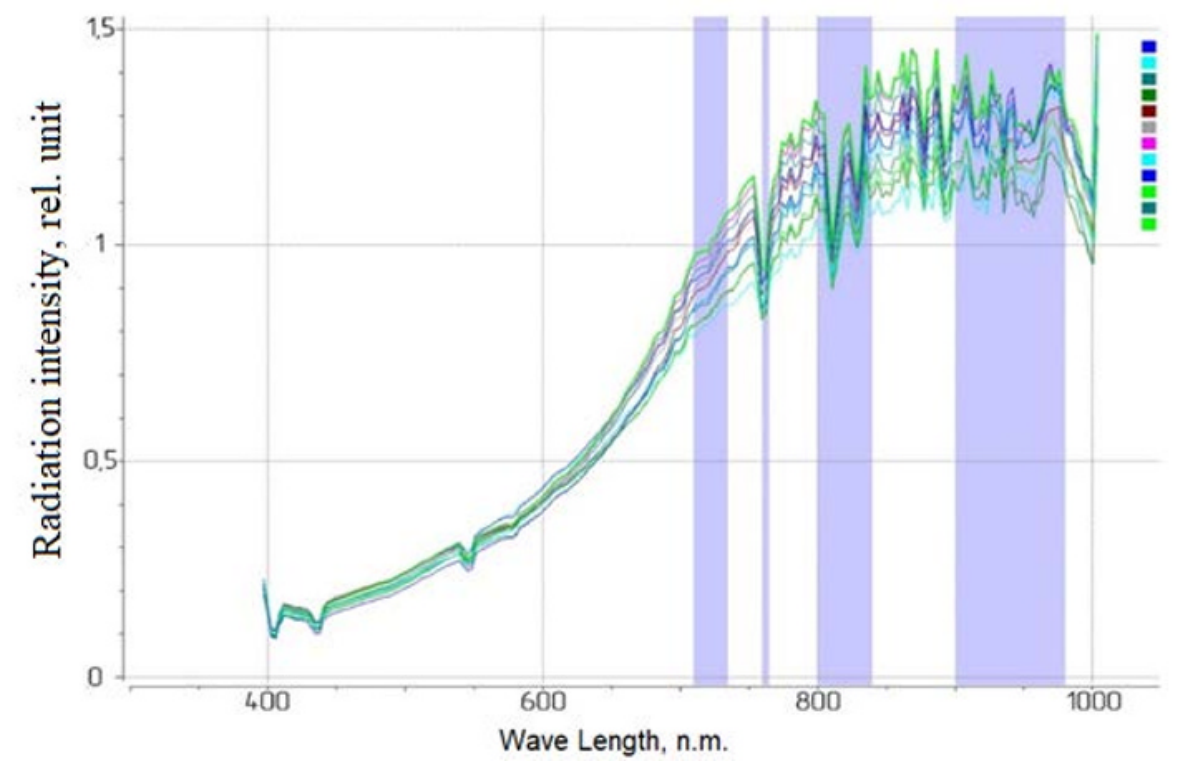

Fig. 4. Specters of healthy samples 


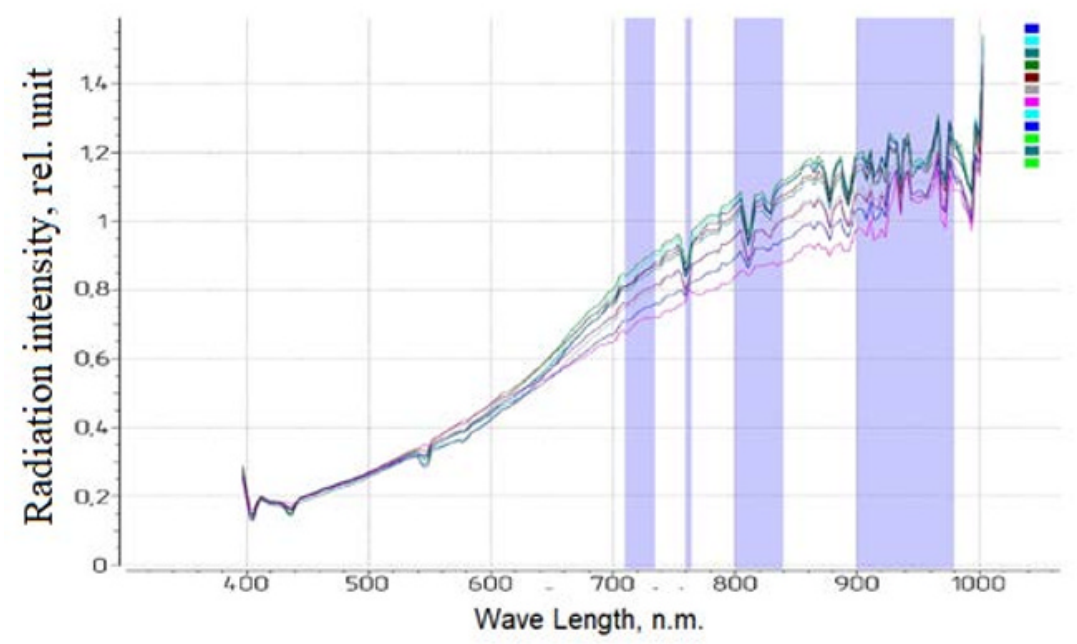

Fig. 5. Specters of infected samples

It can be seen from the graphs that all spectral lines of samples of healthy and infected seeds have common visual points of intensity maxima and minima, which indicates the repeatability of the results.

\section{Results}

After obtaining spectral lines of healthy and infected seeds samples, the operation of averaging and comparison of the results was performed on the general graph shown in Figure. 6.

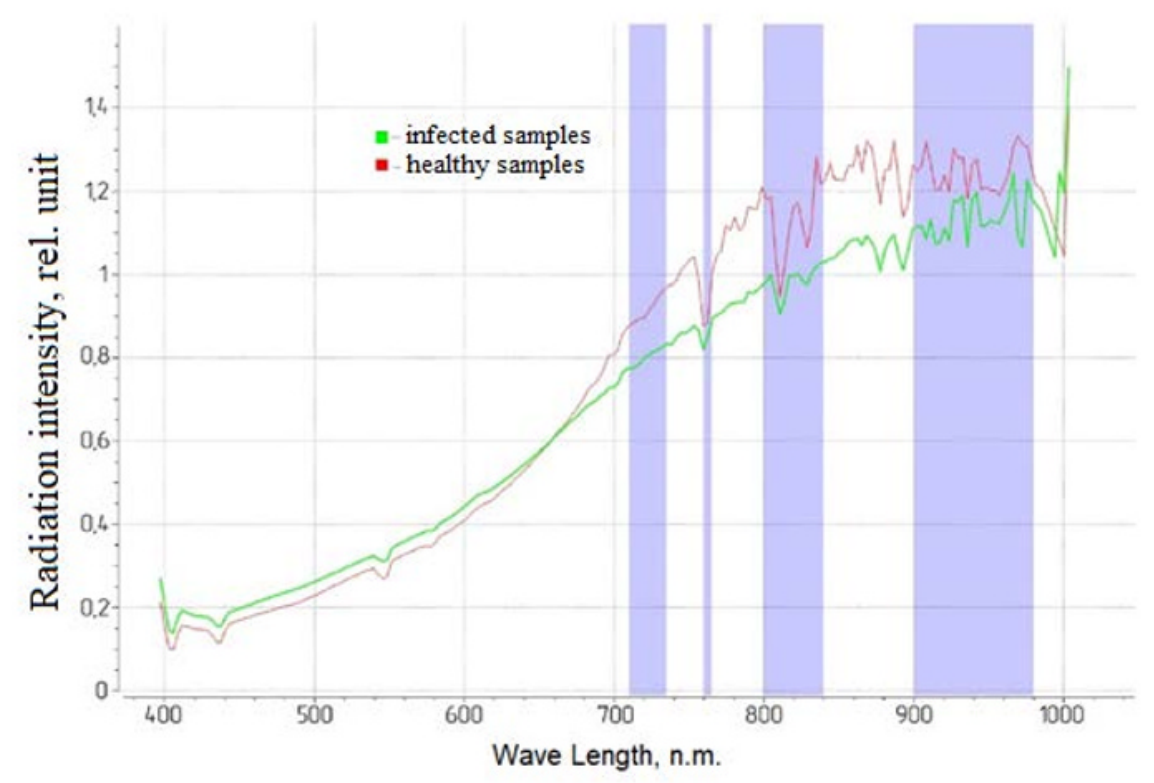

Fig. 6. Results of calculating the specters 
Based on the graphs, the general picture of the effect of fusarium disease on wheat grains can be seen. There is a general decrease in the reflectivity of the grain surface. The strongest deviation of spectral lines is observed in the limit from $660 \mathrm{~nm}$ to $900 \mathrm{~nm}$. In comparison with the healthy sample, the spectral line of infected seeds has a smoother appearance, which indicates the destruction of protein groups as a result of the action of mycotoxins.

\section{Conclusions}

The use of hyperspectral spectroscopy allows non-invasive analysis of biological samples, which in turn simplifies the process of preparing for research. Registration of changes occurs in several broadband spectra from which an image is formed in the form of a hypercube, in which each pixel stores information about wave characteristics.

The differences in the obtained spectra of infected and healthy wheat when normalized to a maximum in the absorption range from $660 \mathrm{~nm}$ to $900 \mathrm{~nm}$ is manifested in much higher absorption in the region of typical protein peaks. This effect is caused by a change in the color of the grain surface, as a result of the appearance of a cobweb-like coating of the fungus mycelium, white or pink, and an accumulation of conidia, in the form of pads. The research was carried out within the framework of agreement No. 075-15-2020-774.

\section{Reference}

1. Bhuvaneswari, K., Fields, P.G., White, N.D.G., Sarkar, A.K., Singh, C.B. and Jayas, D.S. Journal of Stored Products Research 47, 20-24 (2011).

2. Ferreira, D.S., Pallone J.A.L. and Poppi, R.j. Research international 51, 53 - 58 (2013).

3. Ferreira, D.S., Gallo, O.F., Pallone J.A.L. and Poppi, R.j. Food Control 35, 227 232(2014).

4. Kandpal, L.M., Lee S., Kim, M.S., Bae, H. and Cho, B.-K. Food Control 51, 171 - 176 (2015).

5. GOST 12044-93: Agricultural seeds. Methods for determining the incidence of diseases. - M .: STANDARTINFORM. 64 p. (2011)

6. Phytosanitary examination of grain crops (Plant diseases): Recommendations. - M .: FGNU "Rosinformogotech". 140 p. (2002)

7. V.V. Alt, T.A. Gurova, O.V. Elkin, D.N. Klimenko, L.V. Maksimov, I.A. Pestunov, O.A. Dubrovskaya, M.A. Genaev, T.V. Erst, K.A. Genaev, E.G. Komyshev, V.K. Khlestkin, D.A. Afonnikov Genetics and Breeding 24, 259-266 (2020). 